Supplementary Information

\title{
Metal-tagged CRISPR/Cas12a Bioassay Enable Ultrasensitive and Highly Selective Evaluation of Kanamycin Bioaccumulation in Fish Samples
}

Jianyu Hu, ${ }^{+}$Hongjie Song, ${ }^{\ddagger}$ Jing Zhou, ${ }^{+}$Rui Liu, ${ }^{\ddagger}$ and $\mathrm{Yi} \mathrm{Lv}^{\star+\neq}$

+ Analytical \& Testing Center, Sichuan University, Chengdu 610064, PR China

* Key Laboratory of Green Chemistry and Technology of Ministry of Education,

College of Chemistry, Sichuan University, Chengdu 610064, PR China

Email: Ivy@scu.edu.cn

Tel. and Fax: +86-28-8541-2798. 


\section{Table of Content}

Table S1 Oligonucleotides sequence (5' $\left.-3^{\prime}\right)$

Table S2 Operating Conditions of NexION 350 ICP-MS

Table S3 Comparison of aptamer-based assays of Kana detection.

Table S4 Recovery experiment of Kana spike in aquatic samples.

Table S5 Comparison of Kana detection in tissue samples.

Figure S1 Chemical Structure of 1,4,7,10-tetraazacyclododecane-1,4,7-tris-aceticacid10-maleimidoethylacetamide (MMA-DOTA)

Figure S2 ESI-MS spectra of MMA-DOTA $(200 \mu \mathrm{M})$ chelated with $\mathrm{Tm}^{3+}$. (a) Before reaction, (b) after reaction.

Figure S3 “Locked-activated” system recognition of kanamycin.

Figure S4 The secondary structures of (a) Aptamer-1, (b) Aptamer-2 and (c) "Lockedactivated" system.

Figure S5 (a) EDS mapping of original SA-MBs. (b) EDS mapping of SA-MBs captured Tm-Rep.

Figure S6 Native PAGE images of "Locked-activated" system and corresponding nucleic acids.

Figure S7 Optimization of reaction temperature

\section{Reference}


Table S1 Oligonucleotides sequence (5’-3’)

\begin{tabular}{ll}
\hline \hline Activator & CCAAGTCCCAAACACACAAGGGACTCA \\
Aptamer-1 & GGGACTTGGTTTAGGTAATGAGTCCCTTGTGT \\
Aptamer-2 & GTTTGGGACTTGGTTTAGGTAATGAGTCCC \\
crRNA & UAAUUUCUACUAAGUGUAGAUUGAGUCCCUU \\
FAM-Reporter & FAM-AACCGCTTCCCCGACTTCC \\
Tm-Rep & SH C6-TTTATTT-biotin \\
\hline \hline
\end{tabular}

Underline: Kana nucleic acid aptamer sequence 
Table S2 Operating Conditions of NexION 350 ICP-MS

\begin{tabular}{cc}
\hline \hline Conditions & Settings \\
\hline Radiofrequency power & $1300 \mathrm{~W}$ \\
Plasma argon gas flow & $18.00 \mathrm{~L} \mathrm{~min}^{-1}$ \\
Auxiliary argon gas flow & $1.20 \mathrm{~L} \mathrm{~min}^{-1}$ \\
Nebulizer argon gas flow & $0.78 \mathrm{~L} \mathrm{~min}$ min $^{-1}$ \\
Resolution & 2060 \\
Dwell time & $50 \mathrm{~ms}$ \\
Dead time & $35 \mathrm{~ns}$ \\
Sweeps per reading & 120 \\
Isotope monitored & $159 \mathrm{~Tb}$ \\
\hline \hline
\end{tabular}


Table S3 Comparison of aptamer-based assays of Kana detection.

\begin{tabular}{ccccc}
\hline \hline Detection method & Real Sample & Time cost (min) & LOD (nM) & Ref. \\
\hline Colorimetric & Milk & 20 & 5.36 & 1 \\
Fluorescence & Milk, water & 25 & 26.78 & 2 \\
Fluorescence & Milk & 55 & 0.1 & 3 \\
Fluorescence & Milk & 65 & 0.33 & 4 \\
Fluorescence & Serum & 255 & $26 \times 10^{-3}$ & 5 \\
Electrochemistry & Milk & 40 & $0.16 \times 10^{-3}$ & 6 \\
ICPMS & Fish & 30 & $4.06 \times 10^{-3}$ & This work \\
\hline \hline
\end{tabular}


Table S4 Recovery experiment of Kana spike in aquatic samples. 20, 40, 60, 80, 100 pM Kana standard samples were added, respectively.

\begin{tabular}{ccccccc}
\hline \hline \multirow{2}{*}{$\begin{array}{c}\text { Standard } \\
\text { added }\end{array}$} & \multicolumn{2}{c}{ Jialing river } & \multicolumn{2}{c}{ Pseudorasbora parva } & \multicolumn{2}{c}{ Tap water } \\
\cline { 2 - 7 }$(\mathrm{pM})$ & Detection & Recovery & Detection & Recovery & Detection & Recovery \\
\hline 20 & $21.02 \pm 0.92$ & 105.0 & $20.92 \pm 2.54$ & 104.5 & $19.52 \pm 2.04$ & 97.5 \\
40 & $37.24 \pm 1.05$ & 93.0 & $42.14 \pm 1.86$ & 105.3 & $40.84 \pm 4.33$ & 102.0 \\
60 & $59.06 \pm 5.32$ & 98.3 & $49.65 \pm 3.05$ & 82.7 & $62.06 \pm 5.98$ & 103.3 \\
80 & $83.28 \pm 6.73$ & 104.0 & $77.98 \pm 1.75$ & 97.4 & $81.38 \pm 7.03$ & 101.6 \\
100 & $95.60 \pm 8.55$ & 95.5 & $98.30 \pm 2.01$ & 98.2 & $101.90 \pm 7.98$ & 101.8 \\
\hline \hline
\end{tabular}


Table S5 Comparison of Kana detection in tissue samples.

\begin{tabular}{cccc}
\hline \hline Detection method & Real Sample & $\begin{array}{c}\text { Detect limit in } \\
\text { tissue sample } \\
\left(\mu \mathrm{g} \mathrm{g}^{-1}\right)\end{array}$ & Ref. \\
& & 40 & 7 \\
Mass spectrometry imaging & Homogenized tissue & & \\
Solid phase extraction coupled & and bone samples & $6 \times 10^{-3}$ & 8 \\
with HPLC & Soil & & 9 \\
Fluorescent CdTe/CdSe & & & \\
quantum dots & Human serum & 1.0 & This \\
ICPMS & & Fish & work \\
\hline \hline
\end{tabular}


Figure S1 Chemical Structure of 1,4,7,10-tetraazacyclododecane-1,4,7-tris-aceticacid10-maleimidoethylacetamide (MMA-DOTA)<smiles>O=C(O)CN1CCN(CC(=O)O)CCN(CC(=O)O)CCN(CC(=O)O)CC1</smiles> 
Figure S2 ESI-MS spectra of MMA-DOTA $(200 \mu \mathrm{M})$ chelated with $\mathrm{Tm}^{3+}$. (a) Before reaction, (b) after reaction.

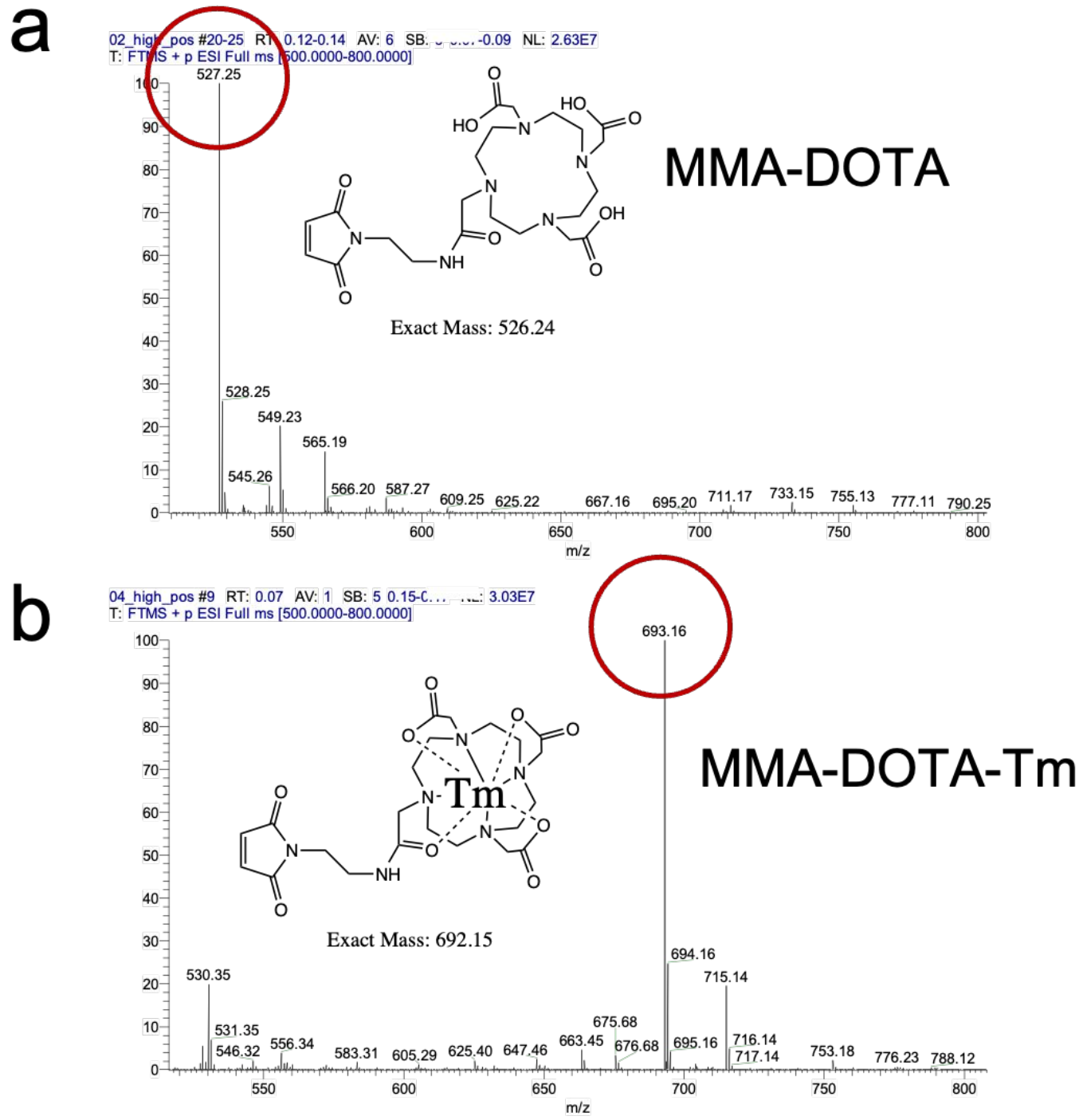


Figure S3 “Locked-activated" system recognition of kanamycin.

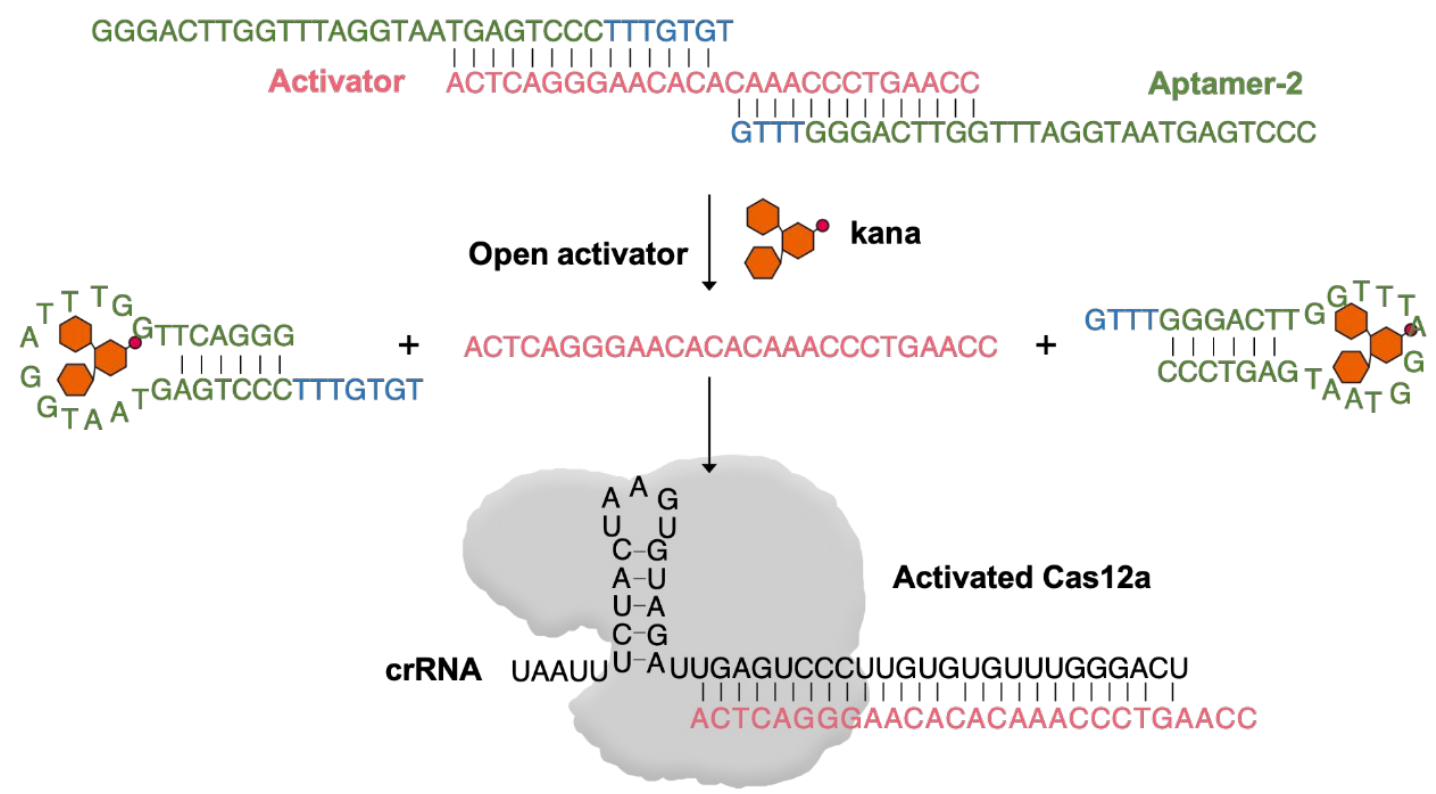


Figure S4 The secondary structures of (a) Aptamer-1, (b) Aptamer-2 and (c) "Lockedactivated" system.

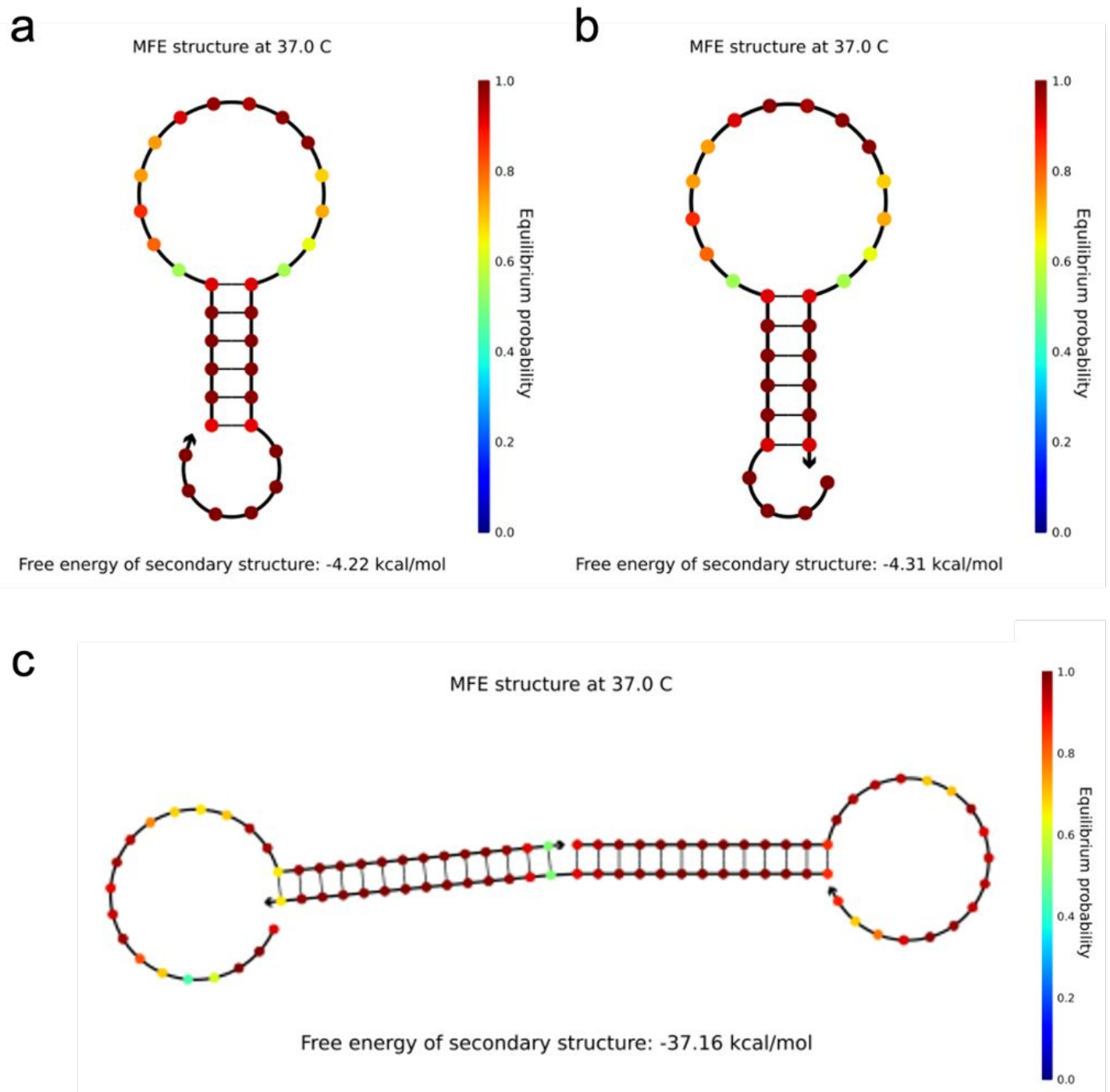


Figure S5 (a) EDS mapping of original SA-MBs. (b) EDS mapping of SA-MBs captured Tm-Rep.

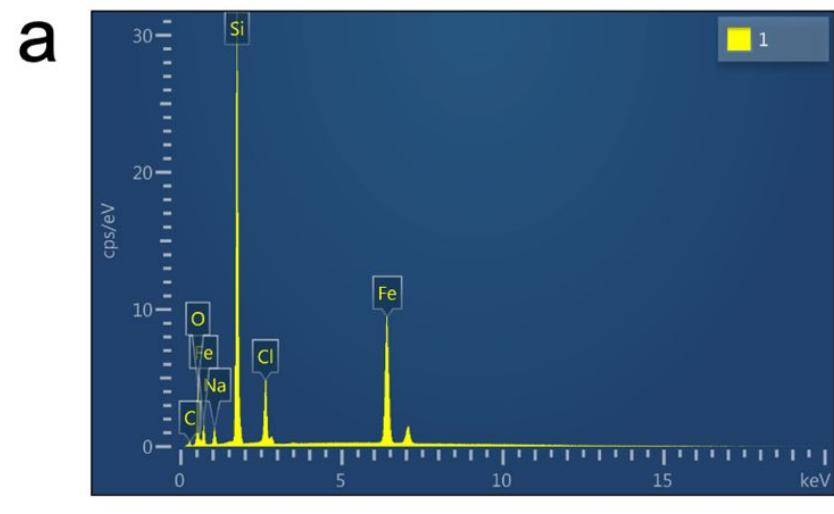

\begin{tabular}{ccc} 
Element & $\mathrm{Wt} \%$ & Atom\% \\
\hline $\mathrm{C}$ & 3.09 & 8.18 \\
$\mathrm{O}$ & 10.64 & 21.18 \\
$\mathrm{Na}$ & 2.26 & 3.13 \\
$\mathrm{Si}$ & 31.12 & 35.27 \\
$\mathrm{Cl}$ & 6.37 & 5.72 \\
$\mathrm{Fe}$ & 46.53 & 26.52 \\
Total & 100.00 & 100.00
\end{tabular}

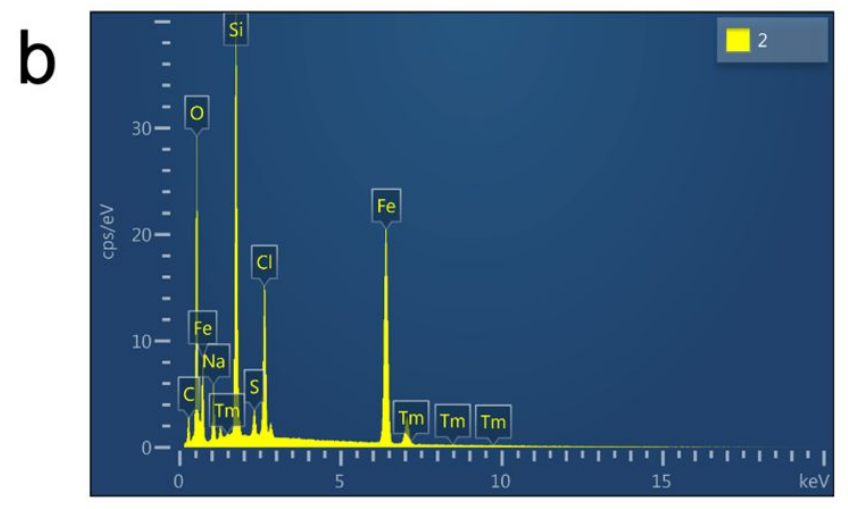

\begin{tabular}{ccc} 
Element & Wt $\%$ & Atom $\%$ \\
\hline $\mathrm{C}$ & 9.76 & 20.48 \\
$\mathrm{O}$ & 24.54 & 38.67 \\
$\mathrm{Na}$ & 3.83 & 4.20 \\
$\mathrm{Si}$ & 14.84 & 13.32 \\
$\mathrm{~S}$ & 1.16 & 0.91 \\
$\mathrm{Cl}$ & 6.99 & 4.97 \\
$\mathrm{Fe}$ & 38.49 & 17.38 \\
$\mathrm{Tm}$ & 0.39 & 0.06 \\
Total & 100.00 & 100.00
\end{tabular}


Figure S6 Native PAGE images of "Locked-activated" system and corresponding nucleic acids.

$\begin{array}{cccc} & \text { Aptamer-1 } & \text { Aptamer-2 } & \text { Activator } \\ 1 & + & - & + \\ 2 & - & + & + \\ 3 & + & + & + \\ 4 & + & - & - \\ 5 & - & + & - \\ 6 & - & - & +\end{array}$

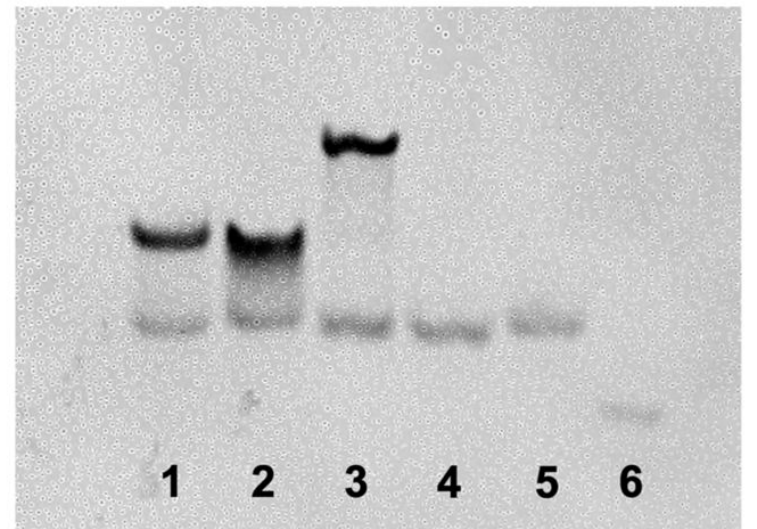


Figure S7 Optimization of reaction temperature

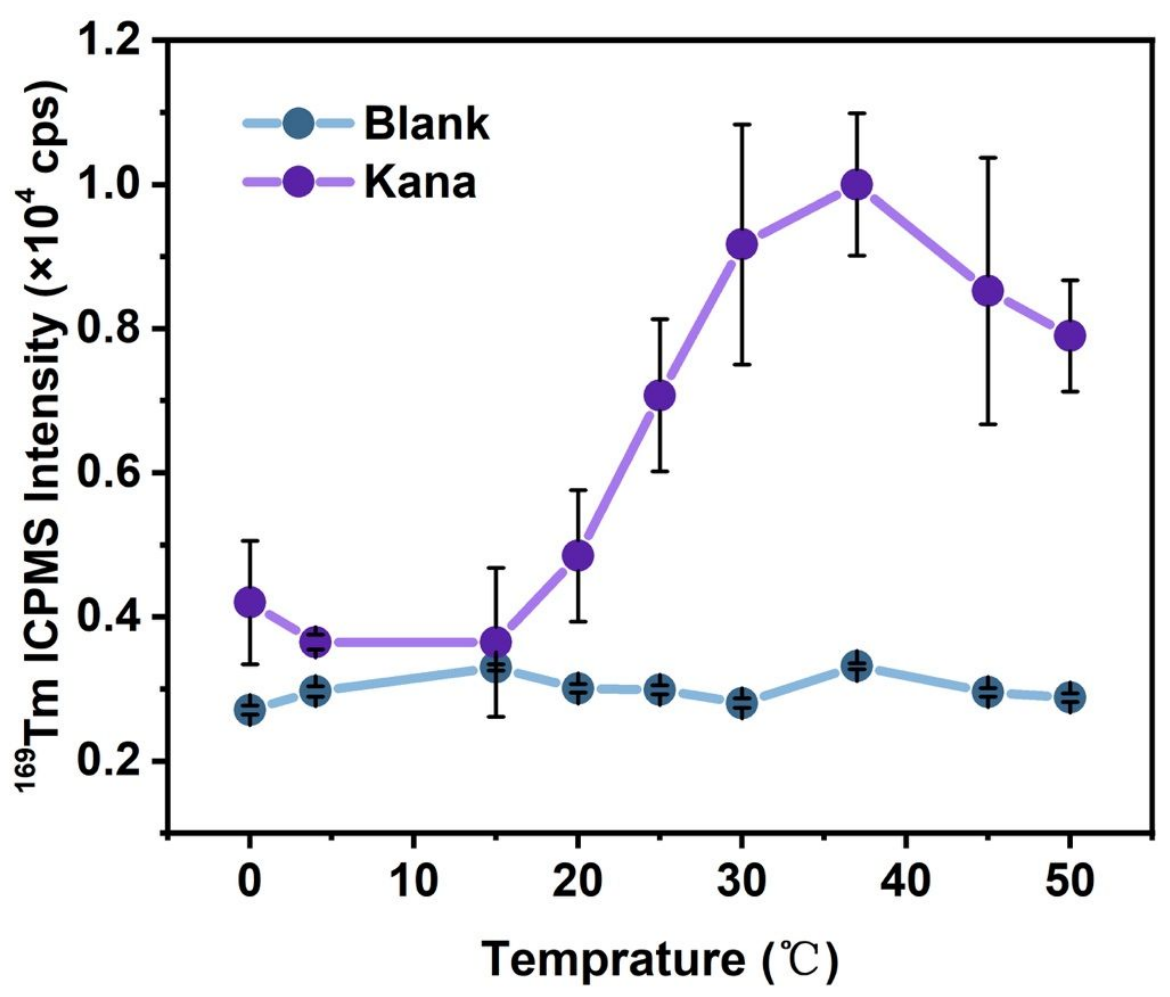




\section{References}

1. Xu, Y.; Han, T.; Li, X.; Sun, L.; Zhang, Y.; Zhang, Y., Colorimetric detection of kanamycin based on analyte-protected silver nanoparticles and aptamer-selective sensing mechanism. Anal. Chim. Acta 2015, 891, 298-303.

2. Geng, Y.; Guo, M.; Tan, J.; Huang, S.; Tang, Y.; Tan, L.; Liang, Y., A fluorescent molecularly imprinted polymer using aptamer as a functional monomer for sensing of kanamycin. Sens. Actuator BChem. 2018, 268, 47-54.

3. Ha, N.-R.; Jung, I.-P.; Kim, S.-H.; Kim, A. R.; Yoon, M.-Y., Paper chip-based colorimetric sensing assay for ultra-sensitive detection of residual kanamycin. Process Biochem. 2017, 62, 161-168.

4. Zhu, Y.; Li, W.; Tan, S.; Chen, T., Label-Free and Simple G-quadruplex-based Turn-Off Fluorescence Assay for the Detection of Kanamycin. Anal. Lett. 2018, 51 (11), 1718-1729.

5. Belal, A. S. F.; Ismail, A.; Elnaggar, M. M.; Belal, T. S., Click chemistry inspired copper sulphide nanoparticle-based fluorescence assay of kanamycin using DNA aptamer. Spectroc. Acta Pt. A-Molec. Biomolec. Spectr. 2018, 205, 48-54.

6. Chen, M.; Gan, N.; Zhou, Y.; Li, T.; Xu, Q.; Cao, Y.; Chen, Y., A novel aptamer- metal ionsnanoscale MOF based electrochemical biocodes for multiple antibiotics detection and signal amplification. Sens. Actuator B-Chem. 2017, 242, 1201-1209.

7. Wang, N.; Dartois, V.; Carter, C. L., An optimized method for the detection and spatial distribution of aminoglycoside and vancomycin antibiotics in tissue sections by mass spectrometry imaging. J. Mass Spectrom. 2021, 56 (3).

8. Sun, Y.; Li, D. G.; He, S.; Liu, P. F.; Hu, Q. B.; Cao, Y. S., Determination and dynamics of kanamycin A residue in soil by HPLC with SPE and precolumn derivatization. Int. J. Environ. Anal. Chem. 2013, 93 (4), 472-481.

9. Mao, Y. Q.; Li, N.; Han, Y.; Zhou, Z. J., CdTe/CdSe quantum dots as a fluorescent probe for kanamycin determination in the pharmaceutical formulation and human serum. Optoelectron. Adv. Mater.Rapid Commun. 2012, 6 (9-10), 879-882. 